\title{
A Cluster of Mutations Disrupt the Avirulence but Not the Virulence Function of AvrPto
}

\author{
Libo Shan, Ping He, Jian-Min Zhou, and Xiaoyan Tang \\ Department of Plant Pathology, Kansas State University, Manhattan 66506-5502, U.S.A. \\ Accepted 7 March 2000.
}

\begin{abstract}
avrPto in Pseudomonas syringae pv. tomato encodes an avirulence protein that triggers race-specific resistance in tomato plants carrying Pto. The AvrPto protein is secreted from $P$. syringae pv. tomato to plant cells through the type III secretion pathway and activates race-specific resistance by a direct interaction with the Pto protein. Here we report that avrPto enhances the virulence of $P$. syringae pv. tomato in a strain-dependent manner in tomato plants lacking Pto. To determine whether the virulence function can be structurally separated from the avirulence function, we examined the virulence activity of a group of AvrPto mutants that carry single amino acid substitutions and lack the avirulence activity on tomato plants. Three mutants that were clustered in the center of AvrPto exhibited virulence activity in tomato plants with or without Pto. The rest of the mutations abolished the virulence. The identification of these mutants suggested that the avirulence function of AvrPto can be structurally separated from the virulence function.
\end{abstract}

Avirulence ( $a v r)$ genes are defined by their ability to induce the hypersensitive response (HR) and disease resistance in host plants containing the corresponding resistance $(R)$ genes (reviewed by Leach and White 1996). The direct or indirect product of an $a v r$ gene serves as the signal that is recognized by the corresponding $R$ gene product, and this recognition subsequently activates the resistance pathway. Pathogens lacking the $a v r$ genes evade the recognition by the host $R$ genes and cause disease.

In addition to the avirulence function, a number of $a v r$ genes have been shown to enhance the virulence of pathogens on plants lacking the corresponding $R$ genes (Kearney and Staskawicz 1990; Swarup et al. 1992; Lorang et al. 1994; Ritter and Dangl 1995; Yang et al. 1996). Mutation of these avr genes reduces the fitness of the pathogens on susceptible hosts by affecting pathogen growth and symptom development. Based on these discoveries, it was postulated that $a v r$ genes were adopted by pathogens as virulence factors but were targeted later by $R$ genes during plant-pathogen coevolution (Collmer 1998).

avrPto was isolated from P. syringae pv. tomato, a pathogen that causes bacterial speck disease on tomato leaves and fruits (Ronald et al. 1992; Salmeron and Staskawicz 1993). It encodes a small, hydrophilic protein of 164 amino acid residues.

Corresponding author: Xiaoyan Tang; E-mail: xtang@plantpath.ksu.edu
avrPto triggers the HR and disease resistance in tomato plants carrying Pto, an $R$ gene that encodes a cytoplasmic serine/ threonine protein kinase (Martin et al. 1993). The AvrPto protein is believed to be injected into the tomato cells by the type III secretion system (Ham et al. 1998). The Pto and AvrPto proteins interact directly in the yeast two-hybrid assay, and the physical interaction between the two proteins determines the gene-for-gene specificity (Scofield et al. 1996; Tang et al. 1996). The interaction between Pto and AvrPto presumably increases the Pto kinase activity, which subsequently activates disease resistance signal transduction pathways through protein phosphorylation.

Recently, we observed that avrPto enhanced the pathogenicity of $P$. syringae pv. tomato in tomato plants lacking the Pto gene. The dual functions of virulence and avirulence of AvrPto raised the following question: how are the two opposite functions related in structure? We have previously identified nine AvrPto point mutations that completely abolished the avirulence activity on tomato plants carrying the Pto gene (X. Tang, L. Shan, V. K. Thara, G. B. Martin, and J. Zhou, unpublished work). These mutants enabled us to determine whether the virulence and the avirulence functions of AvrPto can be structurally separated. We report here that three of the mutants that are tightly clustered in the center of AvrPto maintain the virulence function. The remaining six mutants that are dispersed along the protein disrupt both the virulence and avirulence activities. The three clustered mutations probably define a motif that is required for the avirulence function of AvrPto. Mutations in this motif do not appear to affect the protein architecture or the structure for virulence.

\section{RESULTS}

avrPto enhances the virulence of the $P$. syringae pv. tomato T1 strain.

$\mathrm{T} 1$ is a virulent strain of $P$. syringae pv. tomato that does not contain the avrPto gene. Introduction of the cloned avrPto gene (in the pPtE6 plasmid; Ronald et al. 1992) converts it to an avirulent strain. When inoculated into tomato plants containing the Pto gene (Rio Grande-PtoR, referred to as PtoR), T1(pPtE6) activates disease resistance whereas T1 causes bacterial speck disease (Ronald et al. 1992; Martin et al. 1993). When inoculated into plants lacking Pto (Rio GrandePtoS, referred to as PtoS), both T1 and T1(pPtE6) caused disease. At $10^{6} \mathrm{CFU}$ per $\mathrm{ml}$ or higher concentrations, both $\mathrm{T} 1$ and T1(pPtE6) caused death of the PtoS leaves within 2 days post inoculation, and no significant difference in disease 
A

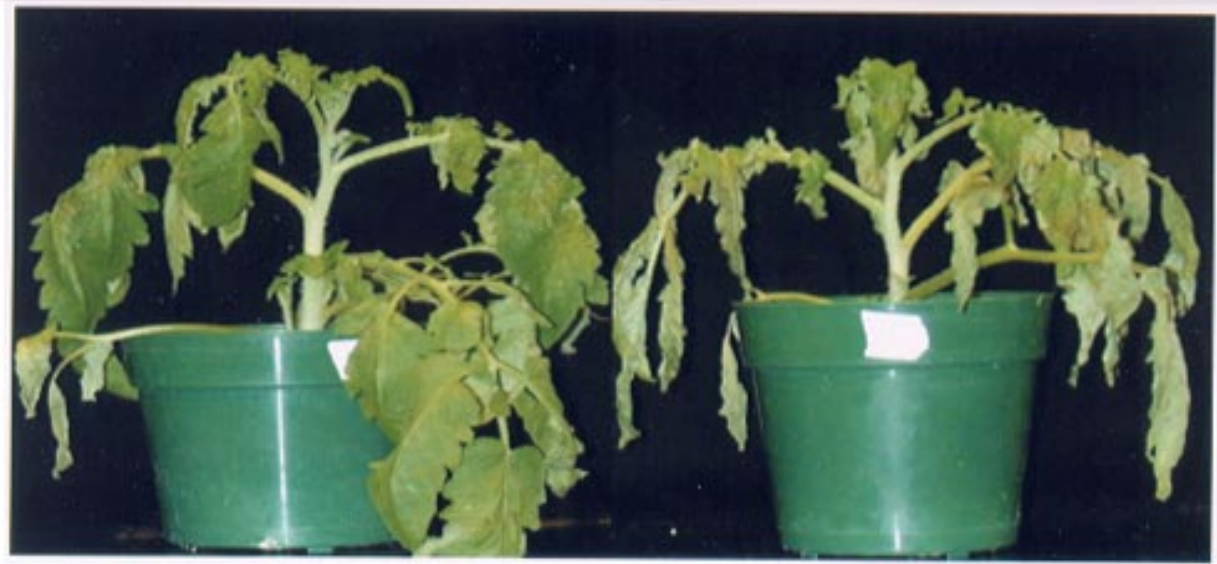

B

\section{T1 T1(pPtE6)}

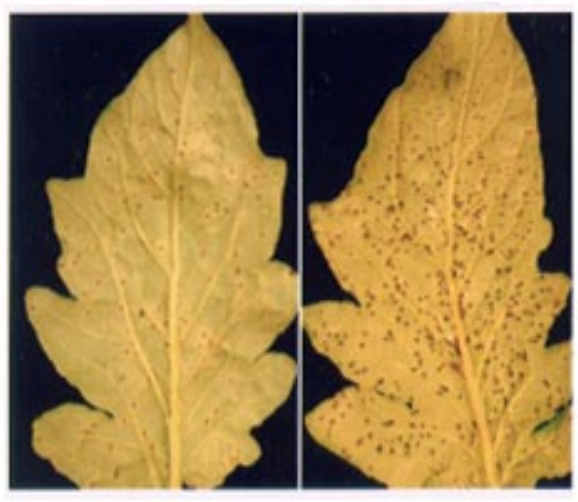

D

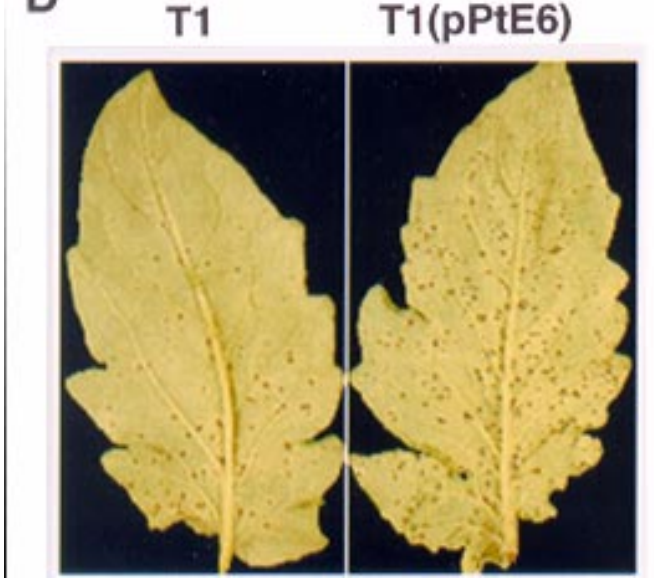

C

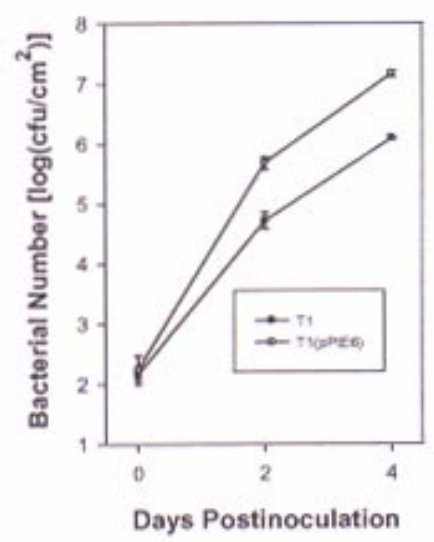

E

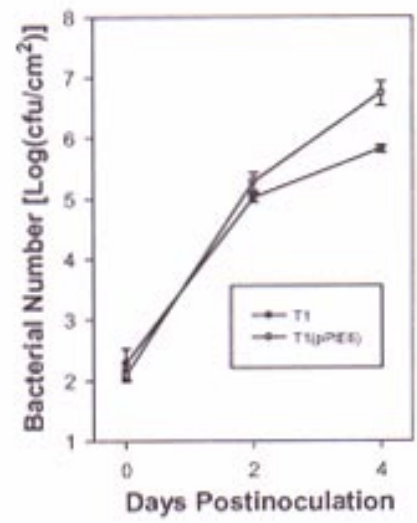

Fig. 1. The virulence function of avrPto in the Pseudomonas syringae pv. tomato T1 strain. A, PtoS plants inoculated with $10^{5} \mathrm{CFU}$ of T1 and T1(pPtE6) per ml. B, PtoS leaves inoculated with $10^{4}$ CFU of T1 and T1(pPtE6) per ml. C, Growth of T1 and T1(pPtE6) in PtoS plants. D, prf-3 leaves inoculated with $10^{4} \mathrm{CFU}$ of T1 and T1(pPtE6) per ml. E, Growth of T1 and T1(pPtE6) in prf-3 mutant. Disease symptoms were documented 4 days post inoculation. For measurement of bacterial growth, tomato plants were inoculated with $10^{4} \mathrm{CFU}$ of T1 and T1(pPtE6) per ml. Six leaf disks $\left(0.5 \mathrm{~cm}^{2}\right.$ in size) from three inoculated plants were divided into three tubes and ground separately. Bacteria were diluted, and plated on King's B plates containing appropriate antibiotics. CFU were counted. Value for each time point represents average of three measurements. Error bars indicate standard error. Similar results were obtained from multiple experiments. 
symptoms caused by these two strains was observed (data not shown). At $10^{5} \mathrm{CFU}$ per ml, both $\mathrm{T} 1$ and $\mathrm{T} 1$ (pPtE6) caused severe disease lesions on PtoS leaves. However, the leaves inoculated with T1(pPtE6) desiccated within 4 days post inoculation, whereas leaves inoculated with $\mathrm{T} 1$ remained alive at this time (Fig. 1A). When the inoculum was further decreased to $10^{4} \mathrm{CFU}$ per $\mathrm{ml}$ or lower, a clear difference in disease symptoms caused by $\mathrm{T} 1$ and $\mathrm{T} 1(\mathrm{pPtE} 6)$ was observed on the inoculated leaves. As shown in Figure 1B, PtoS leaves inoculated with T1(pPtE6) exhibited more disease lesions than leaves inoculated with T1. T1(pPtE6) also multiplied to higher numbers than T1 in PtoS leaves (Fig. 1C). The bacterial numbers reached by $\mathrm{T} 1$ (pPtE6) were about five- to 10-fold higher than those of T1 in PtoS leaves at 4 days post inoculation. A least significant difference statistical test showed that the difference in bacterial growth was significant. Enhanced bacterial growth was consistently observed in numerous experiments (Table 1). Increased bacterial growth closely correlated to greater disease severity in each experiment (data not show). We also inoculated $\mathrm{T} 1$ and $\mathrm{T} 1(\mathrm{pPtE6})$ at the level of $10^{4} \mathrm{CFU}$ per $\mathrm{ml}$ into Money Maker plants, another tomato cultivar that does not contain the Pto gene. As in PtoS plants, T1(pPtE6) multiplied to higher numbers and caused more disease than did strain T1 in Money Maker (data not shown). These results suggest that avrPto enhances the virulence of the T1 strain.

We hypothesize that AvrPto enhances virulence by interacting with the target protein(s) inside the plant cell. When Pto is present, binding of Pto with AvrPto may render AvrPto unavailable to the targets for virulence, and thereby decrease the virulence activity of AvrPto. To test this hypothesis, we inoculated the prf-3 mutant plants with $10^{4} \mathrm{CFU}$ per ml of T1 and T1(pPtE6). The prf-3 mutant has a functional Pto gene. However, the Pto-mediated resistance to T1(pPtE6) is disrupted, because a deletion in the mutant truncates the Prf protein, an essential component of the Pto signal transduction pathway (Salmeron et al. 1996). Four days after inoculation, plants inoculated with both bacterial strains developed a large number of bacterial specks. However, T1(pPtE6) caused more severe symptoms than did T1 (Fig. 1D). T1(pPtE6) also multiplied to significantly higher levels than $\mathrm{T} 1$ in the $p r f-3 \mathrm{mu}-$ tant (Fig. 1E). This result suggests that the presence of Pto alone in the plant cell is unable to block the virulence function of AvrPto. It is possible that the avirulence domain of AvrPto protein is structurally separated from the virulence structure; therefore, binding with Pto does not block the site for the virulence function of AvrPto. It is also possible that Pto does not bind AvrPto in vivo in the absence of Prf protein.

In the natural $P$. syringae pv. tomato isolates DC3000 and PT23, avrPto is a chromosomal gene and exists as a single copy. Deletion of avrPto from the DC3000 and PT23 genomes did not visibly affect the virulence of these two strains (Ronald et al. 1992; Lorang et al. 1994). In T1(pPtE6), however, avrPto was carried by the pPtE6 plasmid with expression controlled by the native promoter of avrPto (Ronald et al. 1992). Although pPtE6 (derived from the pDSK519 plasmid; Ronald et al. 1992) is a low copy plasmid in Pseudomonas spp. (Keen et al. 1988), we could not rule out the possibility that the visible virulence of $\mathrm{T} 1(\mathrm{pPtE6})$ was a result of the increased expression of AvrPto in T1(pPtE6). To test this possibility, we introduced the pPtE6 plasmid into DC3000 and DC30004avrPto and compared the AvrPto protein levels in T1(pPtE6), DC3000, DC3000(pPtE6), and DC3000_avrPto(pPtE6). As shown in Figure 2, AvrPto protein can be detected in both the bacterial cells and the inducing medium for the four strains. The presence of avrPto in pPtE6 plasmid did not lead to a significant increase of AvrPto protein when compared with the chromosomal avrPto gene in DC3000. The detection of AvrPto protein in the inducing medium was consistent with results of others that AvrPto protein is secreted by these strains in the inducing medium (van Dijk et al. 1999). Although it cannot be ruled out that the observed AvrPto protein in the culture medium was partially derived from the lysis of bacterial cells, the results do indicate a similar protein expression level for the DC3000 and T1(pPtE6) strains.

To test whether pPtE6 can enhance the virulence of DC3000, we inoculated DC3000 and DC3000(pPtE6) into PtoS plants and compared the virulence of the two strains. No significant difference was observed in disease symptoms and bacterial growth between the two bacterial strains when tested at a variety of concentrations $\left(10^{3}\right.$ to $10^{4} \mathrm{CFU}$ per ml; Fig. 3). Similarly, we did not detect significant difference in virulence between DC3000_avrPto and DC3000_avrPto(pPtE6) (Fig. 3 ). The presence of avrPto did not affect the virulence of DC3000 on PtoS plants (Fig. 3; Ronald et al. 1992). This is probably related to the high basal virulence of DC3000. At the same inoculation levels, we observed that DC3000 caused more severe diseases on PtoS plants than did the $\mathrm{T} 1$ and T1(pPtE6) strains (data not shown). The minor contribution of avrPto to virulence was probably masked by the high basal virulence of DC3000.

\section{The virulence and avirulence of AvrPto can be separated by point mutations.}

We have identified nine AvrPto mutants with single amino acid substitutions that completely disrupt the interaction with Pto in the yeast two-hybrid system (Fig. 4A). All these mutants lost the avirulence activity on tomato plants carrying the Pto gene (X. Tang, L. Shan, V. K. Thara, G. B. Martin, and J. Zhou, unpublished work). These mutants enabled us to determine whether the virulence and avirulence functions of AvrPto can be structurally separated.

We inoculated the T1 strains carrying individual avrPto mutants into PtoS plants and examined the bacterial population 4 days post inoculation. Three strains carrying avrPto mutant G99V, S94P, and I96T exhibited bacterial growth similar to that of $\mathrm{T} 1$ (pPtE6), and the bacterial numbers of the three strains were significantly higher than that of the T1 strain (Fig. 4B and C). The remaining six avrPto mutants did not result in a significant increase in bacterial growth compared with the T1 strain (Fig. 4B and C). Bacterial growth was closely correlated to disease symptoms caused by these strains (data not shown). We also measured the growth of these mutant strains in PtoR plants. Interestingly, the three strains (G99V, S94P, and I96T) that exhibited higher bacterial numbers in PtoS plants also exhibited higher bacterial numbers than T1 in PtoR plants (Fig. 4C and D). These results indicated that the three AvrPto mutants maintain the virulence function. The three mutations (S94P, I96T, and G99V) are clustered between Ser94 and Gly99, a motif that is required for the tomato Pto recognition (X. Tang, L. Shan, V. K. Thara, G. B. Martin, and J. Zhou, unpublished work). Other mutations are scattered along the AvrPto protein and may affect the 
common structure for both virulence and avirulence or the overall architecture of the protein. Mutations that specifically disrupt avirulence but not virulence suggest that the avirulence and virulence of AvrPto can be structurally separated.

\section{DISCUSSION}

Based on the Potato virus $X$-mediated transient expression of AvrPto that resulted in deleterious effects on a susceptible plant, a virulence function was proposed for the avrPto gene (Tobias et al. 1999). However, avrPto has never been reported

Table 1. Experiments demonstrating the virulence activity of avrPto in Pseudomonas syringae pv. tomato $\mathrm{T} 1$ strain $^{\mathrm{a}}$

\begin{tabular}{|c|c|c|c|c|}
\hline \multirow[b]{2}{*}{ Experiment } & \multicolumn{2}{|c|}{$\begin{array}{l}\text { Bacterial number } \\
(\text { CFU/cm²) }(\text { day 0) }\end{array}$} & \multicolumn{2}{|c|}{$\begin{array}{c}\text { Bacterial number }\left(\times 10^{5}\right) \\
\left(\mathrm{CFU} / \mathrm{cm}^{2}\right)(\text { day } 4)\end{array}$} \\
\hline & T1 & T1(pPtE6) & T1 & T1(pPtE6) \\
\hline 1 & $150^{\mathrm{b}} \pm 21^{\mathrm{c}}$ & $80 \pm 25$ & $12 \pm 3$ & $144 \pm 11$ \\
\hline 2 & $213 \pm 37$ & $217 \pm 3$ & $78 \pm 7$ & $405 \pm 81$ \\
\hline 3 & $283 \pm 18$ & $123 \pm 9$ & $78 \pm 9$ & $401 \pm 83$ \\
\hline 4 & $140 \pm 10$ & $147 \pm 27$ & $122 \pm 23$ & $1036 \pm 249$ \\
\hline 5 & $156 \pm 9$ & $176 \pm 14$ & $52 \pm 15$ & $388 \pm 71$ \\
\hline 6 & $287 \pm 20$ & $247 \pm 29$ & $34 \pm 14$ & $173 \pm 32$ \\
\hline 7 & $73 \pm 28$ & $83 \pm 3$ & $50 \pm 6$ & $160 \pm 15$ \\
\hline
\end{tabular}

${ }^{a}$ Seven experiments were performed at different times to measure bacterial numbers of T1 and T1(pPtE6) in PtoS plants. PtoS plants were inoculated and bacterial numbers measured as described in Materials and Methods.

${ }^{\mathrm{b}}$ Mean of three measurements.

${ }^{\mathrm{c}}$ Standard error.

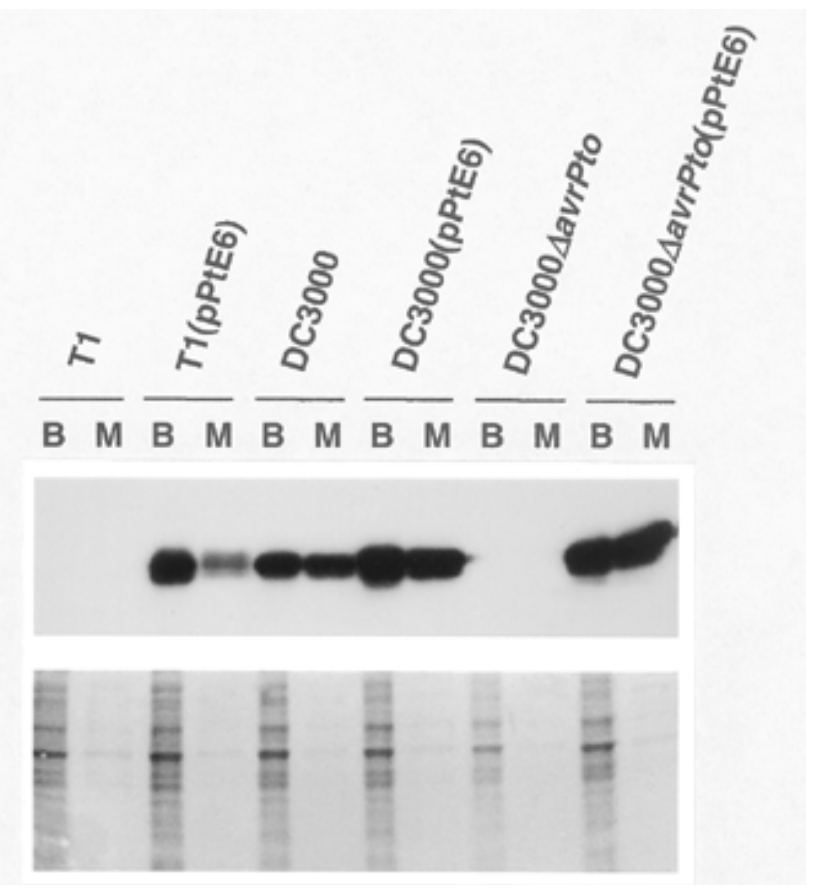

Fig. 2. Expression of AvrPto protein in Pseudomonas syringae pv. tomato strains. Bacteria were separated from inducing medium by centrifugation. Pellet was resuspended in equal volume of distilled water. Presence of AvrPto protein in bacteria (B) and inducing medium (M) was analyzed by anti-AvrPto antibodies. Top, Western blot (immunoblot) analysis of AvrPto protein. Bottom, protein gel-stained with Coomassie blue showing total protein in each sample. to enhance the virulence of $P$. syringae pv. tomato bacteria (Ronald et al. 1992; Lorang et al. 1994). Here we present results showing that avrPto in the P. syringae pv. tomato T1 strain enhanced bacterial growth and disease symptoms in susceptible tomato plants. The discrepancy between our results and previous reports is not immediately understood. It may be that the virulence function was not observed for avrPto because higher levels of bacteria inocula were used in previous reports. Nevertheless, our results were observed in numerous experiments. In addition, we have found that three avrPto mutants with impaired avirulence activity exhibited greater virulence on tomato than the $\mathrm{T} 1$ strain without the avrPto gene. Taken together, these results lead us to conclude that the avrPto gene possesses a detectable virulence function, at least in certain $P$. syringae pv. tomato strains.

Like other $a v r$ genes with demonstrated virulence function (Kearney and Staskawicz 1990; Lorang et al 1994; Ritter and Dangl 1995; Yang et al. 1996), avrPto moderately enhanced the growth of the T1 strain when a small number of bacteria were inoculated into the susceptible plants. This result suggests that avrPto adds virulence to individual $\mathrm{T} 1$ cells. This additional virulence is significant for the aggressiveness of $\mathrm{T} 1$ cells when the initial in planta bacterial population is low. avrPto did not increase the growth and symptom development of the T1 strain when plants were challenged with a high inoculum $\left(>10^{6} \mathrm{CFU}\right.$ per $\left.\mathrm{ml}\right)$ of bacteria. This is probably because, at high bacterial numbers, the contribution of avrPto to virulence is not needed to overcome the plant defense system and establish the parasitic interactions.

The avrPto-mediated virulence appeared to be strain dependent. It was detected reliably in the T1 strain, but was not

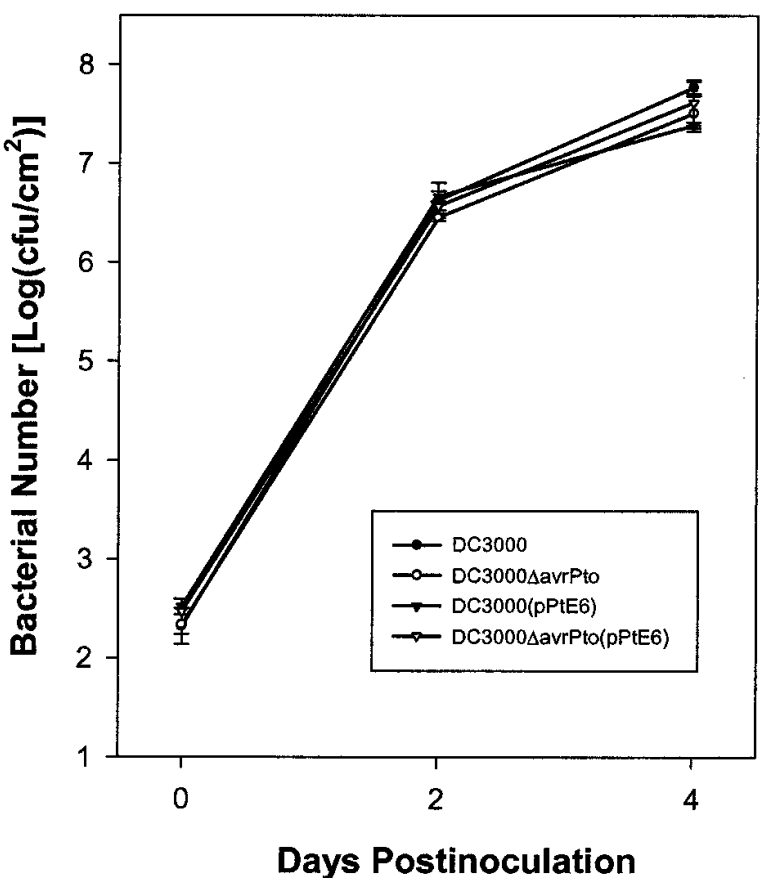

Fig. 3. avrPto does not affect the virulence of DC3000. DC3000, DC3000(pPtE6), DC3000_avrPto, and DC3000_avrPto(pPtE6) $\left(10^{4}\right.$ $\mathrm{CFU} / \mathrm{ml}$ ) were vacuum infiltrated into 6-week-old PtoS plants. Bacterial numbers were measured as described in Figure 1. Error bars indicate standard error. 
detected in DC3000 and PT23, two P. syringae pv. tomato strains carrying a chromosomal avrPto gene. Deletions of avrPto from the DC3000 and PT23 strains were not reported to affect the virulence of these two strains (Ronald et al. 1992; Lorang et al. 1994). Although the avrPto gene used in this study was plasmid borne, the observed virulence was not the result of the plasmid origin, because introduction of pPtE6 into the DC3000 and DC3000 AavrPto strains did not enhance the virulence of these strains on tomato plants. In addition, protein gel-blot analysis indicated that strains carrying pPtE6 produced similar amounts of the AvrPto protein, compared with the DC3000 strain. Thus, the observed virulence activity was not the result of AvrPto overexpression. The straindependent virulence was also reported for the $a v r E$ locus in $P$. syringae pv. tomato. Deletion of avrE apparently decreased the pathogenicity of the PT23 strain but not of DC3000 (Lorang et al. 1994). The redundancy of avrPto in the pathogenicity of DC3000 may be related to other, unidentified

A

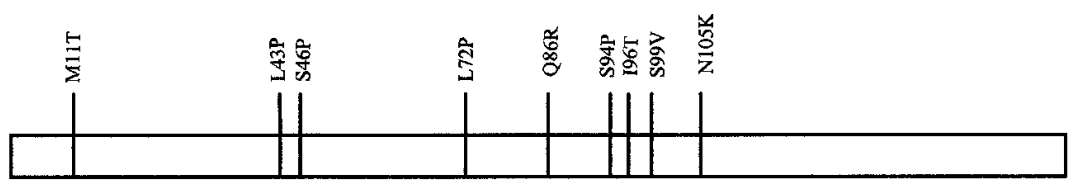

B

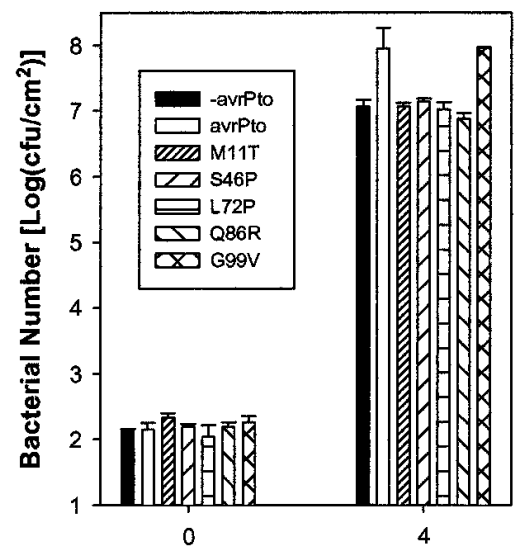

Days postinoculation
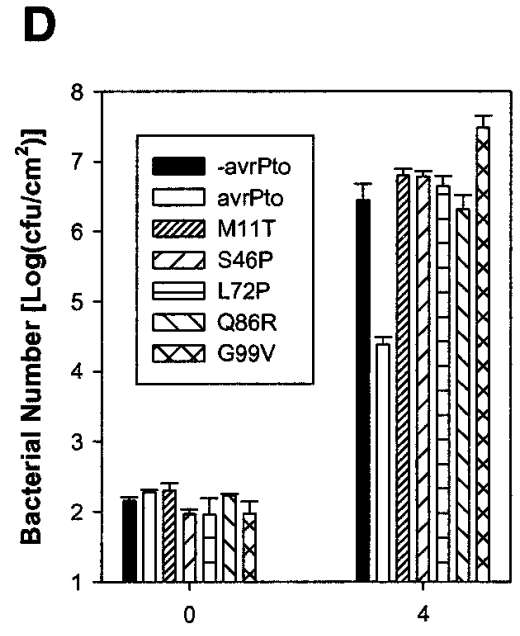

Days Postinoculation
C

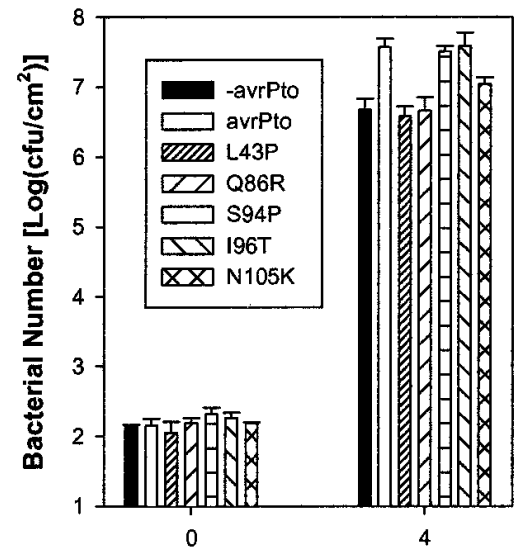

Days Postinoculation

E

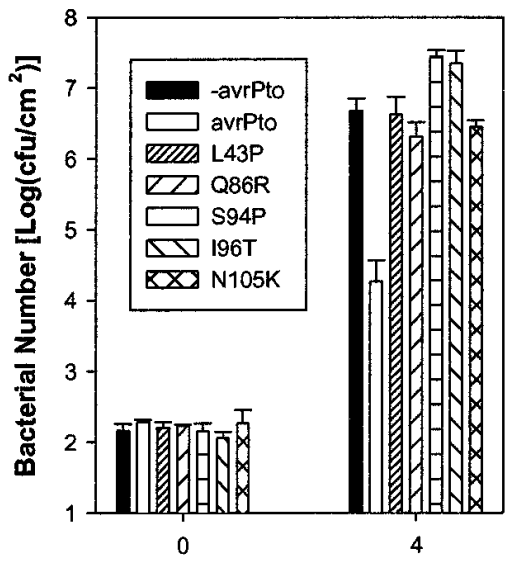

Days Postinoculation

Fig. 4. Maintenance of virulence function in avrPto mutant G99V, S94P, and I96P. A, Position of point mutations in AvrPto protein. AvrPto mutants were named according to the amino acid substitutions. Single letter symbols are used to name amino acids, e.g., M11T means that Met11 in AvrPto is substituted by Thr in the mutant. avrPto mutants were introduced into the T1 strain and the resulting strains $\left(10^{4} \mathrm{CFU} / \mathrm{ml}\right)$ were divided into two groups. Mutant strains in each group were inoculated together with T1 and T1(pPtE6). Bacterial numbers were obtained in the same experiment. Mutant Q86R was included in both groups as a control for reproducibility. Bacterial numbers were measured as described in Materials and Methods. B and C, Bacterial numbers in PtoS plants. D and E, Bacterial numbers in PtoR plants. Value in each time point represents average of three measurements. Error bars indicate standard error. 
virulence factors in this strain. We have observed that DC3000 strains with or without avrPto were more aggressive on PtoS plants than the T1 and T1(pPtE6) strains. This suggested that DC3000 has a high basal virulence, and the high basal virulence probably masked the minor virulence contribution of avrPto. The virulence function of avrPto may be detectable in DC3000 when other virulence factors are deleted.

One actively sought strategy to identify durable resistance genes is to target virulence factors that play an important role in the fitness of the pathogen (Laugé et al. 1998). This is based on the hypothesis that any mutations in the important virulence genes that evade detection by the corresponding $R$ genes impose a penalty on the pathogen's survival. However, our studies with the AvrPto mutants suggest that this strategy may not guarantee durable resistance. We have previously identified a central motif in AvrPto between Ser94 and Gly99 that is required for recognition by the tomato Pto. Mutations in this motif completely abolished the AvrPto-Pto interaction and the avirulence activity of AvrPto in the tomato plant (X. Tang, L. Shan, V. K. Thara, G. B. Martin, and J. Zhou, unpublished work). However, the same mutations in this motif had no effect on the virulence activity. These results suggest that pathogens can adapt to the deployment of the $R$ gene without sacrificing virulence. A greater understanding of the structural bases for the avirulence and virulence activity is needed for the successful identification of durable resistance genes. Only $R$ genes that target the structure critical for virulence are likely to provide a durable resistance.

\section{MATERIALS AND METHODS}

Plant inoculation and measurement of bacterial growth.

The tomato cultivars Rio Grande-PtoS and Rio GrandePtoR were grown in a greenhouse. Six-week-old plants were used for bacterial inoculation. The $P$. syringae pv. tomato strains containing avrPto and avrPto mutants were grown in a King's B (KB) medium containing $100 \mathrm{mg}$ of rifampicin and $25 \mathrm{mg}$ of kanamycin per liter (Tang et al. 1999). The P. syringae pv. tomato strains that do not carry avrPto were grown in $\mathrm{KB}$ medium with $100 \mathrm{mg}$ of rifampicin per liter. Bacteria were grown overnight in liquid $\mathrm{KB}$ medium with the appropriate antibiotics. The bacterial culture was washed twice with 10 $\mathrm{mM} \mathrm{MgCl}$ and resuspended in $10 \mathrm{mM} \mathrm{MgCl}{ }_{2}$. Bacterial density was determined by absorbance at $\mathrm{OD}_{600}$, and diluted to the desired concentrations in $10 \mathrm{mM} \mathrm{MgCl}_{2}$ plus $0.04 \%$ Silwet L-77 (OSI, Danbury, CT). Tomato plants were inoculated by vacuum infiltration and kept in the greenhouse. For leaf bacteria measurement, six leaf disks $\left(0.5 \mathrm{~cm}^{2}\right.$ in size) were collected from three inoculated plants and divided into three tubes. Each tube contains two leaf disks. The leaf disks were ground in $10 \mathrm{mM} \mathrm{MgCl} 2$. Bacteria were diluted and plated on appropriate $\mathrm{KB}$ plates. $\mathrm{CFU}$ were counted. The number for each time point represents the average of three measurements.

\section{Detection of AvrPto protein.}

avrPto cDNA was cloned in-frame into the pGEX-KG vector for the production of GST-AvrPto fusion protein in Escherichia coli (Guan and Dixon 1991). The GST-AvrPto fusion was purified with glutathione-conjugated cellulose beads (Sigma, St. Louis, MO), and the fusion protein was cleaved as described (Zhou et al. 1995). The AvrPto protein was further purified with sodium dodecyl sulfate-polyacrylamide gel electrophoresis (SDS-PAGE) and was used as an antigen to raise polyclonal antibodies. Anti-AvrPto antibodies were purified from the crude antiserum as described (Sambrook et al. 1989). Briefly, the purified AvrPto protein was immobilized on a polyvinylidene difluoride (PVDF) membrane (Millipore, Bedford, MA) and incubated with the crude anti-AvrPto antiserum. The membrane was then washed extensively and the antibodies were eluted.

To examine AvrPto production in Pseudomonas spp., the bacterial strains were grown overnight in $\mathrm{KB}$ medium with appropriate antibiotics. The bacteria were washed twice with the minimum medium (Huynh et al. 1989), diluted with the same medium to $10^{8} \mathrm{CFU}$ per $\mathrm{ml}$, and grown for 1 additional day. Bacteria were separated from medium by centrifugation and resuspended in the same amount of distilled water. Equal volumes of medium and of bacterial suspension were boiled in $1 \times$ protein sample buffer (Sambrook et al. 1989). Proteins were separated by SDS-PAGE, transferred to PVDF membrane, and analyzed by Western blotting (immunoblotting) with anti-AvrPto antibodies according to standard procedures (Sambrook et al. 1989). Membrane-bound, secondary antibodies were visualized by ECL Western Blotting Detection Reagents (Amersham Life Science, Buckinghamshire, England) and X-ray film (Kodak, Rochester, NY).

\section{Introduction of avrPto and avrPto mutants into strains of $P$. syringae pv. tomato.}

Mutations on avrPto that abolished the interaction with Pto were identified by the yeast two-hybrid screening (Tang et al. 1996) and confirmed by sequencing analysis. Plasmid pPtE6 (DSK519::avrPto; Ronald et al. 1992) was used as the vector for cloning of avrPto mutants into the pDSK519 plasmid. pPtE6 was digested with BamHI and $\mathrm{XbaI}$, and the wild-type avrPto sequence was replaced by the mutant avrPto. Because the BamHI site is in the coding region of avrPto, the expression of avrPto mutants in Pseudomonas spp. was under the control of the native avrPto promoter. The avrPto mutants in pDSK519 were introduced into T1 by tri-parental mating and selected on KB plates containing $100 \mathrm{mg}$ of rifampicin and 25 $\mathrm{mg}$ of kanamycin per liter. The presence of avrPto mutants in the T1 strain was confirmed by DNA blot analysis.

\section{ACKNOWLEDGMENTS}

We thank Brian Staskawicz for providing us the prf-3 mutant. We are grateful to Jan Leach, Frank White, Randy Warren, and Thara Venkapatta for the critical reading of the manuscript. X. T. is supported by the Kansas State University Plant Biotechnology Center and a USDA grant (no. 9802511). Contribution number 00-242-J.

\section{LITERATURE CITED}

Collmer, A. 1998. Determinants of pathogenicity and avirulence in plant pathogenic bacteria. Curr. Opin. Plant Biol. 1:329-335.

Guan, K.-L., and Dixon, J. E. 1991. Eukaryotic proteins expressed in Escherichia coli: An improved thrombin cleavage and purification of fusion proteins with glutathione S-transferase. Anal. Biochem. 192: 262-267.

Ham, J. H., Bauer, D. W., Fouts, D. E., and Collmer, A. 1998. A cloned Erwinia chrysanthemi Hrp (type III protein secretion) system functions in Escherichia coli to deliver Pseudomonas syringae Avr signals 
to plant cells and to secrete Avr proteins in culture. Proc. Natl. Acad. Sci. USA 95:10206-10211

Huynh, T. V., Dahlbeck, D., and Staskawicz, B. J. 1989. Bacterial blight of soybean: Regulation of a pathogen gene determining host cultivar specificity. Science 245:1374-1377.

Kearney, B., and Staskawicz, B. J. 1990. Widespread distribution and fitness contribution of Xanthomonas campestris avirulence gene avrBs2. Nature 346:385-386.

Keen, N. T., Tamaki, S., Kobayashi, D., and Trollinger, D. 1988. Improved broad-host-range plasmids for DNA cloning in gram-negative bacteria. Gene 70:191-196.

Laugé, R., Joosten, M. H. A. J., Haanstra, J. P. W., Goodwin, P. H., Lindhout, P., and De Wit, P. J. G. M. 1998. Successful search for a resistance gene in tomato targeted against a virulence factor of a fungal pathogen. Proc. Natl. Acad. Sci. USA 95:9014-9018.

Leach, J. E., and White, F. F. 1996. Bacterial avirulence genes. Annu. Rev. Phytopathol. 34:153-179.

Lorang, J. M., Shen, H., Kobayashi, D., Coosey, D., and Keen, N. T. 1994. avrA and avrE in Pseudomonas syringae pv. tomato PT23 play a role in virulence on tomato plants. Mol. Plant-Microbe Interact. 7: 508-515.

Martin, G. B., Brommonschenkel, S., Chunwongse, J., Frary, A., Ganal, M. W., Spivey, R., Wu, T., Earle, E. D., and Tanksley, S. D. 1993. Map-based cloning of a protein kinase gene conferring disease resistance in tomato. Science 262:1432-1436.

Ritter, C., and Dangl, J. L. 1995. The avrRpml gene of Pseudomonas syringae pv. maculicola is required for virulence on Arabidopsis. Mol. Plant-Microbe Interact. 8:444-453.

Ronald, P. C., Salmeron, J. M., Carland, F. M., and Staskawicz, B. J. 1992. The cloned avirulence gene avrPto induces disease resistance in tomato cultivars containing the Pto resistance gene. J. Bacteriol. 174: 1604-1611.

Salmeron, J. M., Oldroyd, G. E. D., Rommens, C. M. T., Scofield, S. R., Kim, H. -S., Lavelle, D. T., Dahlbeck, D., and Staskawicz, B. J. 1996. Tomato $\mathrm{Prf}$ is a member of the leucine-rich-repeat class of plant disease resistance genes and lies embedded within the Pto kinase gene cluster. Cell 86:123-133.

Salmeron, J. M., and Staskawicz, B. J. 1993. Molecular characterization and $h r p$ dependence of the avirulence gene avrPto from Pseudomonas syringae pv. tomato. Mol. Gen. Genet. 239:6-16.

Sambrook, J., Fritsch, E. F., and Maniatis, T. A. 1989. Molecular Cloning: A Laboratory Manual. 2nd ed. Cold Spring Harbor Laboratory, Cold Spring Harbor, NY.

Scofield, S. R., Tobias, C. M., Rathjen, J. P., Chang, J. H., Lavelle, D. T., Michelmore, R. W., and Staskawicz, B. J. 1996. Molecular basis of gene-for-gene specificity in bacterial speck disease of tomato. Science 274:2063-2065.

Swarup, S., Yang, Y., Kingsley, M. T., and Gabriel, D. W. 1992. A Xanthomonas citri pathogenicity gene, pthA, pleiotropically encodes gratuitous avirulence on nonhosts. Mol. Plant-Microbe Interact. 5:204213.

Tang, X., Frederick, R. D., Zhou, J., Halterman, D. A., Jia, Y., and Martin, G. B. 1996. Initiation of plant disease resistance by physical interaction of AvrPto and the Pto kinase. Science 274:2060-2063.

Tang, X., Xie, M., Kim, Y. J., Zhou, J., Klessig, D. F., and Martin, G. B. 1999. Overexpression of Pto activates defense responses and confers broad resistance. Plant Cell 11:15-29.

Tobias, C. M., Oldroyd, G. E., Chang, J. H., and Staskawicz, B. J. 1999. Plants expressing the Pto disease resistance gene confer resistance to recombinant PVX containing the avirulence gene avrPto. Plant J. 17: 41-50.

van Dijk, K., Fouts, D. E., Rehm, A. H., Hill, A. R., Collmer, A., and Alfano, J. R. 1999. The Avr (effector) proteins HrmA (HopPsyA) and AvrPto are secreted in culture from Pseudomonas syringae pathovars via the Hrp (type III) protein secretion system in a temperature- and pH-sensitive manner. J. Bacteriol. 181:4790-4797.

Yang, Y., Yuan, Q., and Gabriel, D. W. 1996. Watersoaking function(s) of XcmH1005 are redundantly encoded by members of the Xanthomonas avr/pth gene family Mol. Plant-Microbe Interact. 9:105-113.

Zhou, J., Loh, Y.-T., Bressan, R. A., and Martin, G. B. 1995. The tomato gene Ptil encodes a serine/threonine kinase that is phosphorylated by Pto and is involved in the hypersensitive response. Cell 83:925-935. 